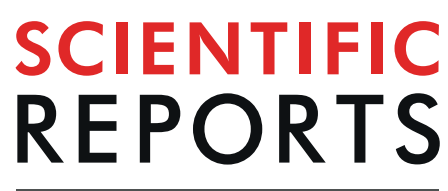

natureresearch

\title{
The Relation between Alpha/Beta Oscillations and the Encoding of Sentence induced Contextual Information
}

\author{
René Terporten ${ }^{1,2^{*}}$, Jan-Mathijs Schoffelen ${ }^{1}{ }^{1}$, Bohan Dai ${ }^{1,2}$, Peter Hagoort ${ }^{1,2}$ \& \\ Anne Kösem $(10)^{1,2,3}$
}

Pre-stimulus alpha $(8-12 \mathrm{~Hz})$ and beta $(16-20 \mathrm{~Hz})$ oscillations have been frequently linked to the prediction of upcoming sensory input. Do these frequency bands serve as a neural marker of linguistic prediction as well? We hypothesized that if pre-stimulus alpha and beta oscillations index language predictions, their power should monotonically relate to the degree of predictability of incoming words based on past context. We expected that the more predictable the last word of a sentence, the stronger the alpha and beta power modulation. To test this, we measured neural responses with magnetoencephalography of healthy individuals during exposure to a set of linguistically matched sentences featuring three levels of sentence context constraint (high, medium and low constraint). We observed fluctuations in alpha and beta power before last word onset, and modulations in M400 amplitude after last word onset. The M400 amplitude was monotonically related to the degree of context constraint, with a high constraining context resulting in the strongest amplitude decrease. In contrast, pre-stimulus alpha and beta power decreased more strongly for intermediate constraints, followed by high and low constraints. Therefore, unlike the M400, pre-stimulus alpha and beta dynamics were not indexing the degree of word predictability from sentence context.

Sentence level language comprehension results from dynamic cognitive processes which combine and unify smaller linguistic units to create meaning ${ }^{1-6}$. These cognitive processes occur online, while the sentence unfolds, instantiating unified meaning which relates to the computation of semantics, spanning the whole utterance. During this process, a context representation is compared and updated on a moment to moment basis. The bias provided by the momentarily established context alters subsequent linguistic processing ${ }^{7-9}$. One classical approach to investigate the impact of linguistic predictions at the neuronal level is to measure the N400 component, which is called the M400 in magnetoencephalographic (MEG) studies ${ }^{10-12}$. The N400 functionally marks how surprising the occurrence of a target word is provided the past sentential context ${ }^{13}$. The N400 amplitude is stronger for anomalous or unexpected items, but importantly it is also influenced by how constraining the preceding sentential context is (i.e. how predictive are subsequent linguistic items based on past context), such that the N400 amplitude increases with lower sentence context constraints ${ }^{11,14-18}$. While not being linked to predictive processes directly, these N400 modulations suggests that sentence context constraints alter predictions that are encoded prior to target word occurrence.

Prediction in its minimal sense can be understood as changes in brain states in response to contextual information which facilitate the processing of new input ${ }^{19,20}$. Recent evidence suggests that neural rhythmic activity could be involved in the prediction of linguistic input during sentence processing. Neural oscillatory responses have been linked to the $\mathrm{N} 400$ and semantic predictions, such as theta $(4-7 \mathrm{~Hz})$ oscillations ${ }^{21,22}$ and gamma band $(>40 \mathrm{~Hz})$ activity ${ }^{23-26}$. Importantly however, theta and gamma oscillatory activity has mostly been observed as neural markers for semantic predictions after target word presentation. In contrast, and to the interest of the present study, other brain oscillatory responses in the alpha $(8-12 \mathrm{~Hz})$ and beta $(16-20 \mathrm{~Hz})$ frequency ranges

${ }^{1}$ Max Planck Institute for Psycholinguistics, Wundtlaan 1, 6525 XD, Nijmegen, The Netherlands. ${ }^{2}$ Donders Centre for Cognitive Neuroimaging, Kapittelweg 29, 6525 EN, Nijmegen, The Netherlands. ${ }^{3}$ Lyon Neuroscience Research Center (CRNL), Brain Dynamics and Cognition Team, INSERM U1028, CNRS UMR5292, Université Claude Bernard Lyon 1, UdL, Lyon, France. *email: rene.terporten@mpi.nl 


\begin{tabular}{|l|l|}
\hline \multicolumn{2}{|l|}{ Stimulus material examples } \\
\hline Condition & Stimulus \\
\hline \multirow{2}{*}{ HC } & (NL) Op dit gebouw heb je een goed uitzicht. \\
\cline { 2 - 2 } & (EN) On this building you got a good view. \\
\hline \multirow{2}{*}{ MC } & (NL) Op deze toren heb je een goed uitzicht. \\
\cline { 2 - 2 } & (EN) On this tower you got a good view. \\
\hline \multirow{2}{*}{ LC } & (NL) In deze wijk heb je een goed uitzicht. \\
\cline { 2 - 2 } & (EN) In this area you got a good view. \\
\hline
\end{tabular}

Table 1. Example Dutch sentence triplet from the final stimulus set with its English translation. The context constraining conditions were manipulated by changing one context constraining word.

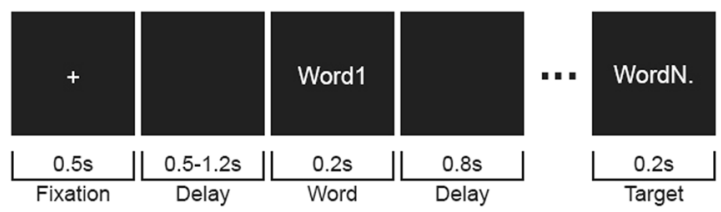

Figure 1. A schematic display of a trial procedure. A trial began with the display of a fixation period, followed by a blank screen. Subsequently the sentence was visually displayed by a word by word presentation, up to the final word as indexed by the period. Between words, a black screen served as delay before a subsequent word was shown.

have been linked to both domain-general and linguistic predictive mechanisms prior to the apparition of a target word. Alpha and beta oscillations are hypothesized to reflect a domain-general mechanism for the prediction of upcoming sensory input $\mathrm{t}^{27,28}$ and to constitute top-down mechanisms that shape the communication of sensory information between distant neural networks ${ }^{29-31}$. Beyond sensory processing, recent theory and evidence suggests that alpha and beta oscillations would also be involved in linguistic prediction ${ }^{19,23,28}$. Decreases in alpha power $r^{21,25,32,33}$ and low-beta power ${ }^{25,33,34}$ have previously been linked to the processing of sentential context constraints. Specifically, the power decrease has been found to be stronger when sentential context is highly predictive of the last word of the sentence than when the prediction of the last word cannot be made based on past context $^{21,25,32-37}$. The power decrease has been explained to reflect stronger engagement of the brain areas of the language network in scenarios in which predictions can be formed from past sentential context ${ }^{25,38}$. Yet, the evidence for alpha and beta oscillations being involved in language prediction is still debated, as it has only been observed between extreme situations, i.e. between very predictable sentences or completely unpredictable sentences ${ }^{21,25,33}$. If alpha and beta power reflect the degree of predictability of an upcoming word, we hypothesized that alpha/beta power should gradually decrease with higher context constraint.

To test this, we presented multiple graded groups of sentences context constraints. Participants passively read sentences belonging to either a low (LC), medium (MC) or high (HC) context constraining condition (Table 1). Crucially, we constructed the sentence material using triplets of sentences, and modulating the context constrain by only changing one word in the sentence (see Methods for details). Neuronal activity was measured online using MEG, before and after display of a target word. Based on previous findings ${ }^{21,25}$, we predicted that pre-stimulus alpha and beta power would differ between different conditions of predictability. The power decrease is expected to be strongest for the HC, followed by the MC and LC condition (Fig. 1).

\section{Materials \& Methods}

Participants. In total, thirty-five students (mean age 24 years, range $18-43 ; 16$ males) took part in the experiment. All participants provided their informed consent in accordance with the declaration of Helsinki, and the local ethics committee (CMO region Arnhem-Nijmegen). All experimental protocols were approved by the review board of the Donders Centre for Cognitive Neuroimaging prior to the start of the experiment. The participants were all Dutch native speakers, right-handed, had normal or corrected-to-normal vision and none of them suffered from neurological impairment or dyslexia. Two participants were excluded because they did not finish the experiment. Therefore, thirty-three participants were included for further analyses (mean age 24 years, range $18-43 ; 15$ males).

Stimulus material. The stimulus set consisted of 253 sentence triplets, including 203 critical and 50 filler sentence triplets. Each sentence within a critical triplet belonged to either a high context (HC), medium context (MC), or low context (LC) constraining condition. The different degree of constraint within a triplet was achieved by manipulating only one word, the context constraining word, which was always at the same position within a sentence with regard to a triplet (Table 1$)$. Across the conditions, these context constraining words were matched with regard to word length $(\mathrm{F}(2,606)=0.784, \mathrm{p}=0.457$, with a Mean (SE) of HC: 7.12 (2.26); MC: 7.1 (2.54); LC: $7.37(2.61))$ and word frequency $(\mathrm{F}(2,584)=1.984, \mathrm{p}=0.138$, with Mean (SE) of HC: 2.4 (0.78); MC: 2.56 (0.87); LC: 2.5 (0.84); based on the Dutch SUBTLEX-NL database ${ }^{40}$. The stimuli were pretested in a sentence completion 
task in order to verify the step-like degree of context constraints within a triplet (from high, to medium, to low). For this task - independent from the MEG experiment - a sample of participants $(\mathrm{N}=51)$ were required to complete a sentence presented on a computer screen, for which the final word was missing. Participants performed the experiment with one of three counterbalanced lists. Each list included the same number of critical sentences from either of the three context constraining conditions. The validation of the conditions was performed in two subsequent steps: first, the degree of context constraint per sentence was evaluated by calculating the percentage of participants that would finish a sentence with the same word. These probabilities are thought to reflect the degree to which the sentential context biases subsequent language processing. As expected, HC sentences resulted in the highest percentage of participants proposing the same word as cloze (Mean (SE) $=77 \%(17.74)$ ), followed by MC (Mean (SE) $=50 \%(18.67)$ ) and LC (Mean (SE) $=28 \%(11.97)$ ). The three conditions differed significantly from each other with regard to their degree of context constraints $(\mathrm{F}(2,606)=442.842, \mathrm{p}<0.001)$.

Second, and in order to create the final stimulus set, the final word from the HC sentences with the highest percentage was chosen as sentence ending for all sentences within a triplet. This approach resulted in cloze probabilities for the final word - the target word - that were different from the percentages of the context constraints for the MC and LC conditions. Still, the cloze probabilities differed significantly between conditions $(\mathrm{F}(2$, $606)=468.155, \mathrm{p}<0.001$ ), with HC showing the highest cloze probability (Mean (SE) $=77 \%(17.74)$ ), followed by MC $($ Mean $(\mathrm{SE})=42 \%(25.94))$ and LC (Mean $(\mathrm{SE})=15 \%(15.82))$. The cloze probabilities are thought to reflect how surprising the final word occurrence is, given the past sentential context. In our stimulus set, context constraint measures were highly correlated with measures of cloze probability $(r=0.93, p<0.001)$.

In the MEG experiment, participants were presented with one of the counterbalanced lists, with an additional set of 50 filler sentences. The filler sentences did not differ between lists but followed a different sentence structure as compared to the critical sentences.

Experimental procedure. Participants were comfortably seated in a dimly illuminated and magnetically shielded room. All participants were instructed to place their arms on the arm rest of the chair, with access to a button box with their right hand. In front of each participant, at a distance of $80 \mathrm{~cm}$ and with a $25^{\circ}-35^{\circ}$ viewing angle, a screen was located on which all stimulus material was displayed. The words were shown in black, on a grey background. Participants were instructed to silently read the displayed sentences on the screen, and to focus on the content of each sentence. Furthermore, it was highlighted that sometimes (after $20 \%$ of the sentences; subjects were not informed about the precise percentage) a question would be asked about the content of the previous displayed sentence. The participants were then required to answer this question with 'yes' or 'no' by button press. The answer possibilities ('yes'/'no') were randomly displayed on the left or right side of the screen and a left or right button had to be pressed accordingly. These question trials were catch trials, intended to ensure that participants were actively processing the meaning of the sentences, without focusing their attention on the contextual constraints. A trial started with the display of a fixation cross in the middle of the screen for $500 \mathrm{~ms}$. The fixation cross was followed by a blank screen for a random interval of 500-1200 ms. Subsequently, the word-by-word presentation of the sentence began, with each word being displayed for $200 \mathrm{~ms}$, followed by a blank screen of $800 \mathrm{~ms}$. An interval of $1000 \mathrm{~ms}$ was chosen in order to record pre-stimulus alpha and beta activity that is not influenced by the evoked response to the previous displayed word. After a sentence ended, another blank screen occurred for $2000 \mathrm{~ms}$ (Fig. 1). After that, either a catch question was displayed, with the whole question in the middle of the screen and the yes-no answers randomly split to the left or right side, or the next trial began. In total, participants read 253 sentences ( 253 trials) in random order, which came from one of three lists, counterbalanced on the three levels of context constraints. The total amount of trials was divided into four blocks, separated by small breaks in-between. The length of a break was self-determined by the participants and the task could be continued by button press. In total, the data acquisition lasted $60 \mathrm{~min}$.

Data acquisition. All data were acquired using a 275 axial gradiometers CTF Omega MEG system. Horizontal and vertical bipolar electrooculography (EOG) as well as electrocardiography (ECG) were recorded in order to discard eye blinks, eye movements and heart beats contaminated trials. All electrophysiological signals were low-pass filtered at $300 \mathrm{~Hz}$, digitized at $1200 \mathrm{~Hz}$, and stored for off-line analysis. Three coils were placed on the nasion and the left and right ear canal to register the head position with respect to the gradiometers. The coils enabled real-time monitoring of the head position throughout the experiment ${ }^{41}$. Next to the MEG recordings, magnetic resonance images (MRIs) were obtained from 32 of the participants with a $1.5 \mathrm{~T}$ or $3.0 \mathrm{~T}$ Siemens system. By means of attached markers at the same anatomical locations as the head coils, the MRIs could be aligned to the MEG coordinate system.

Data preprocessing. All data were analyzed using the open-source Matlab toolbox Fieldtrip ${ }^{42}$. From the MEG data, a time-window of interest was segmented $2 \mathrm{~s}$ before and after the onset of a sentence's final word for each trial. This segmentation therefore included the blank delay period just before onset of the target word, where the effect of context constraints is expected to occur, and the period after onset of the target word. The segmented data were low-pass filtered at $150 \mathrm{~Hz}$. The $50 \mathrm{~Hz}$ line noise components were removed by using a notch filter. Artifact identification and rejection was done in three steps. First, MEG jump and muscle artifacts were identified by visual inspection of amplitude variance over trials. Second, artifacts related to eye-movements and cardiac activity were identified and removed by means of an independent component analysis (fastICA) ${ }^{43}$, followed by backprojection. The independent components were visually inspected and removed from the sensor data, if they resembled heartbeat, eye-movements or blinks (as compared to the recorded EOG and ECG). Third, the resulting data were again visually inspected to remove any remaining artifacts. From this procedure, on average $11 \%$ of trials and $1.5 \%$ of MEG sensors were excluded from further analysis. 
Event-Related Field (ERF) analysis. Event-related fields were investigated to observe M400 modulations after the last word onset. This correlate is the magnetic counterpart of the classical N400 measured by electroencephalography and inhabits the same time-course and response properties ${ }^{44,45}$. For each condition, the epochs were first low-pass filtered at $35 \mathrm{~Hz}$. All ERFs were then baseline corrected based on a time window of -300 ms $-0 \mathrm{~ms}$ relative to target word onset. To facilitate comparison across participants the ERFs were transformed to a combined synthetic planar gradient representation ${ }^{46}$. The M400 component was calculated by averaging over the planar gradient field amplitude from $250 \mathrm{~ms}$ to $600 \mathrm{~ms}$ following target word onset.

Time-frequency analysis. Time-frequency analysis was first done for the time-window of $-800 \mathrm{~ms}$ to $0 \mathrm{~ms}$ relative to the sentence's target word onset, including only the blank delay period. Additionally, the M400 sensitive time-window after target word onset was considered for time-frequency analyses, including a window from $200 \mathrm{~ms}$ up to $700 \mathrm{~ms}$. Alpha and beta power were estimated for each condition using fast Fourier transform for a frequency range of $8 \mathrm{~Hz}$ to $12 \mathrm{~Hz}$ for the alpha, and $16 \mathrm{~Hz}$ to $20 \mathrm{~Hz}$ for the beta frequency bands (based on Wang et al. ${ }^{25}$ ), with a Hanning-tapered $500 \mathrm{~ms}$ sliding window in time steps of $10 \mathrm{~ms}$. No baseline correction was performed on the time-frequency data, statistics were performed by contrasting individual conditions of context constraints. Time-frequency plots were created using a wider frequency range of $2 \mathrm{~Hz}$ to $30 \mathrm{~Hz}$ (using a fixed $500 \mathrm{~ms}$ sliding Hanning window in time steps of $10 \mathrm{~ms}$ and frequency steps of $2 \mathrm{~Hz}$ ) for a time window of -1000 $\mathrm{ms}$ to $1000 \mathrm{~ms}$. Power was averaged over channel clusters that were a result of the statistical comparison between conditions for the alpha and beta frequency band respectively.

Source analysis. To estimate the sources of the oscillatory activity, the Dynamical Imaging of Coherent Sources (DICS) beamforming approach was applied to the data ${ }^{47}$. The volume conduction model was constructed from the individual anatomical MRI as a single shell representation of the inside of the skull. This model was used to compute the forward model according to Nolte ${ }^{48}$. The initial co-registration between the headmodel and MEG sensors was achieved by manually identifying the anatomical landmarks of the nasion and two auricular fiducials, and was additionally refined, using the subject-specific three-dimensional digitised representation of the scalp, as obtained by a Polhemus digitizer. The source space was discretized into a three dimensional grid with a $6 \mathrm{~mm}$ resolution. Source reconstruction was performed using a spatial filter, which was computed by combining the cross-spectral density (CSD) matrices from all three conditions (HC, MC, LC). The CSDs were computed using the Fast Fourier transform of the data with multitapering, with a center frequency of $10 \mathrm{~Hz}$ or $18 \mathrm{~Hz}$ for the alpha (averaged over the time window 540-0 ms, relative to target word onset) and beta (averaged over 450-0 ms) frequency band respectively. All visualizations are based on interpolated data onto the MNI template. The different conditions of the source reconstructed data were compared based on cluster-based permutation statistics as described below.

Cluster-Based permutation statistics. Statistical evaluation was done using non-parametric cluster-based permutation tests ${ }^{49}$. First, we computed F-statistics to quantify the effect of context constraints (three levels: HC, MC, LC) for each sensor and time point. These F-statistics were used to define the clusters for the non-parametric statistical testing: clusters consisted of samples whose F-values were above threshold (threshold: F-value associated with a p-value of 5\%) and were adjacent in space and time. Cluster-level statistics were computed by taking the sum of F-values within each cluster. The distribution of the cluster-level statistics under the null hypothesis was obtained by repeating this procedure for 5000 permutations of random relabeling of the conditions. Clusters whose test-statistics fell in the highest 5 th percentile of its reference distribution were considered significant.

\section{Results}

Behavioral performance. In order to confirm the participant's attention to the experimental task, their performance was measured during catch trials that occurred after presentation of $20 \%$ of the sentences. The overall accuracy measures show a mean ceiling performance of $95 \%(\mathrm{SE}=4.65), 96 \%(\mathrm{SE}=4.42)$ and $97 \%(\mathrm{SE}=2.83)$ for the HC, MC and LC sentences respectively. There were no significant differences in accuracy with respect to the different conditions (Accuracy: $\mathrm{F}(2,31)=0.474, \mathrm{p}=0.627$ ). This indicates that the participants were paying attention to the content of the presented sentences.

Event-Related fields after target word onset. In this experiment, participants read words preceded by a context with different degrees of constraint (three context-constraint conditions: high, medium and low context constraints) while brain signals were recorded online. We first analyzed the effect of context constraints on the event-related fields upon target word presentation. Based on previous literature ${ }^{11,14}$ we expected a monotonous relationship between cloze probability and the M400 component. Consistently, as can be seen from the amplitude fluctuations of the event-related activity (Fig. 2), amplitude differences between the three conditions emerged within the typical M400 time-window. The M400 amplitude strength decreased with increasing cloze probability, such that the HC condition displayed the lowest M400 amplitude, followed by the MC and LC conditions. The cluster-based statistics revealed a main effect of context constraints on the M400 amplitude strength in a pre-defined time window of $250 \mathrm{~ms}$ to $600 \mathrm{~ms}$ after target word onset; this effect was most pronounced over a left-frontal localized cluster of sensors (Fig. 2, cluster $p=0.005$ ). The post-hoc contrasts (based on pairwise T-tests) revealed that the effect was mainly driven by a difference between $\mathrm{HC}$ vs. $\mathrm{LC}(p<0.001)$ and HC vs. MC $(p=0.013)$. Although the M400 amplitude was smaller for MC than for LC condition, this difference was not significant. These effects were overall in line with the current literature showing that the M400 amplitude reflects semantic retrieval and unification of the target word with the preceding context. 


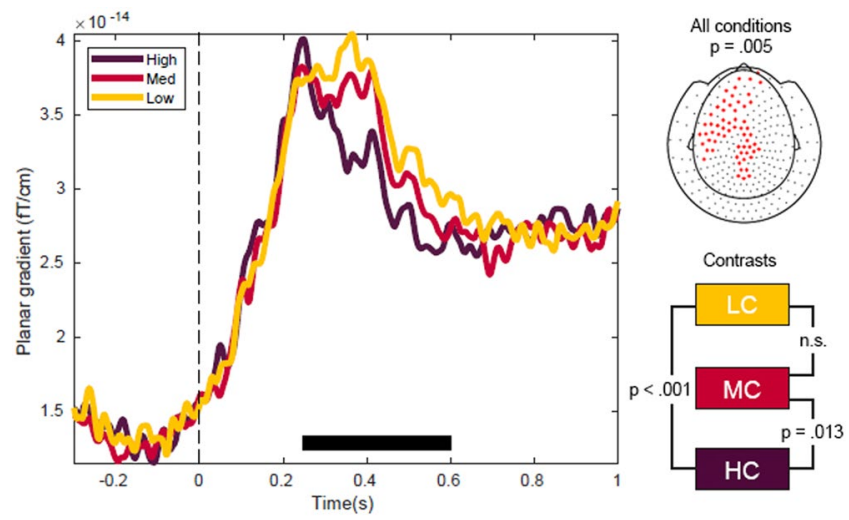

Figure 2. The event related fields of the M400 component at target word onset as averaged across channel clusters. The M400 amplitude is gradually modulated by the degree of context constraints, resulting in the lowest amplitude in HC, followed by MC and LC. The black horizontal line in the main figure highlights the time window [ $250 \mathrm{~ms}, 600 \mathrm{~ms}$ ] pre-defined for the spatial cluster-based permutation test. The upper right topography shows the MEG sensors that formed a cluster as result from the statistical comparison of the conditions. The effect is most pronounced over left lateralized sensors.

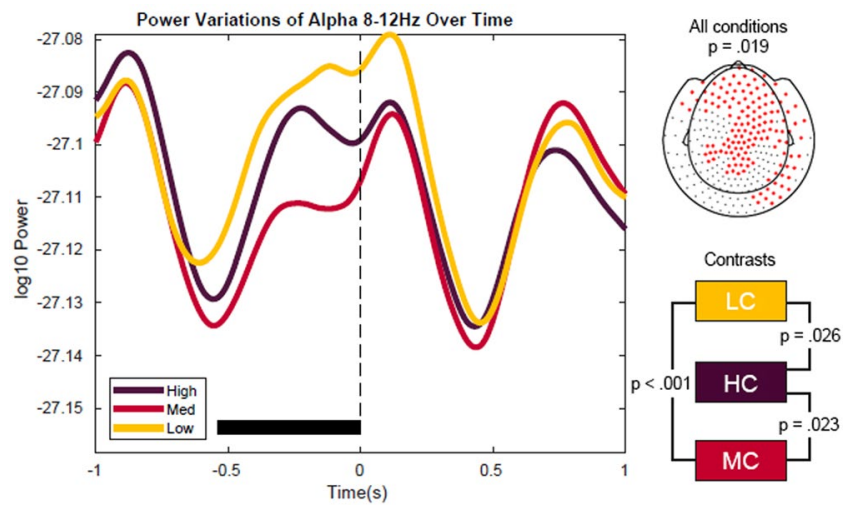

Figure 3. Alpha power fluctuations vary as a function of context constraint before target word onset as averaged across channel clusters. The black horizontal line in the main figure highlights the time window $[-540$ $\mathrm{ms}, 0 \mathrm{~ms}]$ of the spatio-temporal cluster for which the difference between conditions was significant. The upper right topography shows the MEG sensors of the cluster. The effect of context constrains is most pronounced over frontal and posterior sensors. Over these sensors, alpha power showed the strongest decrease for the MC condition, followed by HC and LC.

Alpha/Beta power modulations before target word onset. Next, we investigated the effect of content constraints on alpha and beta power modulations before target word onset. Based on earlier research ${ }^{25}$, an effect was suggested to occur during the delay period, just before the display of the target word. The cluster-based statistics for the alpha $(8-12 \mathrm{~Hz})$ frequency band revealed a significant difference between all three conditions with regard to power $(p=0.019)$ for a time-window of $-540 \mathrm{~ms}$ to $0 \mathrm{~ms}$ relative to target word onset. This effect was most pronounced over a widespread set of sensors, including anterior, central and posterior sensors (Fig. 3). Over these sensors, alpha power showed the strongest decrease for the MC condition, followed by HC and LC (Fig. 3). The post-hoc contrasts of these conditions indicate that the power decrease is significantly different between HC vs. MC $(p=0.023)$, MC vs. LC $(p<0.001)$, and HC vs. LC. $(p=0.026)$. Post-hoc comparisons of power (averaged over $\mathrm{t}($ alpha $)=-540-0 \mathrm{~ms}$ and $\mathrm{t}($ beta $)=-450 \mathrm{~ms}-0 \mathrm{~ms})$ with respect to the whole spectrum $(2 \mathrm{~Hz}$ to $30 \mathrm{~Hz})$ showed that the strongest difference between context constraints peaks in the lower alpha range ( $8 \mathrm{~Hz}$, Fig. 4).

We performed source reconstruction to allow for a more detailed description of the brain areas involved in the observed sensor-level effect. Source-level statistical evaluation indicated that this effect was most pronounced in parietal areas, with a bias to the right hemisphere (Fig. 5, $\mathrm{p}=0.003$, cluster-based corrected).

The sensor-level analyses in the beta $(16-20 \mathrm{~Hz})$ frequency band revealed a similar tendency as for the alpha results, though the effects were not significant (cluster with lowest p-value in cluster-based corrected statistics: $\mathrm{p}=0.077$, see Figs. 6 and 7).

Source statistics in turn indicated a significant F-contrast across all conditions with the effect being most pronounced over a set of frontal and parietal areas, biased to left frontal cortex (Fig. 8, p =0.002, cluster-based corrected). The power fluctuations were, similar to the results on alpha power, non-monotonically related to each 

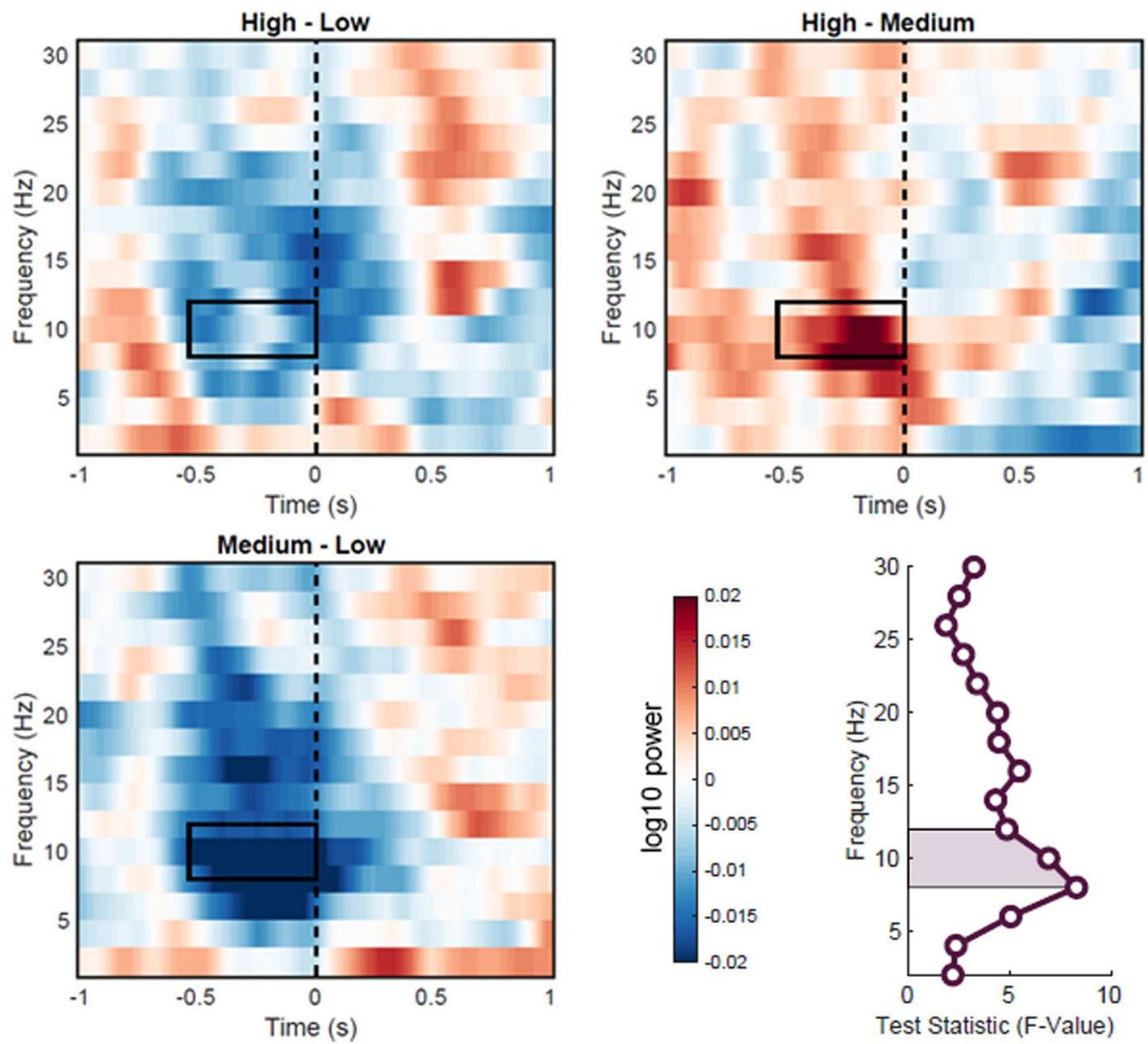

Figure 4. Time-frequency power contrasts between context constraint conditions within the alpha band sensor cluster. Time-frequency plots indicate power as averaged across sensors that were identified by the cluster-based permutation statistics of the alpha frequency band $(8-12 \mathrm{~Hz})$. The dashed line marks the onset of the target word. The rectangle indicates the time-window of the respective cluster, and alpha frequency band. Lower right figure shows the F statistics of the main effect of context constraints across frequencies, averaged within the time window $[-540 \mathrm{~ms}, 0 \mathrm{~ms}]$. The area under the curve indicates the pre-defined alpha frequency range used for cluster based analysis $(8-12 \mathrm{~Hz})$. The effect of context constraint is stronger in the lower alpha range $(8 \mathrm{~Hz})$.

other. The MC condition was again displaying the strongest decrease followed by HC and then the LC condition (Figs. 6, 7).

Alpha/Beta power modulations after target word onset. To cover any oscillatory effects within the M400 sensitive time-window, alpha and beta power modulations were investigated as a function of context constraints after target word onset. The individual cluster-based statistics for both frequency bands revealed no significant difference between the three conditions with regard to power, within this particular time-window.

\section{Discussion}

The current study investigated the role of pre-stimulus alpha and beta oscillations as a neural marker for sentence context constraints. Our results confirm the sensitivity of pre-stimulus alpha and beta power to different levels of constraint. Power of alpha and beta decreases when sentential context constraints are high as compared to when the context constraints are low, in line with previous findings ${ }^{21,25,33}$. However, we report the strongest decrease in alpha and beta power for the medium context constrain conditions. The data contradicts our initial hypothesis and instead indicate that pre-stimulus alpha and beta oscillations are non-monotically related to the amount of contextual constraint which in turn reflects the predictability of the sentence ending. In line with earlier findings, the M/N400 amplitude was monotonically modulated by the degree of constraint, resulting in the lowest amplitude for high, followed by medium and low context constraints. The results suggest that pre-stimulus alpha and beta oscillations and the M/N400 component are neural markers that relate to distinct processes during sentence context evaluation.

In agreement with classic findings, our results show the M/N400 magnitude monotonically decreases with increasing context constraints, and this finding can be taken as support for M/N400 integration and/or 


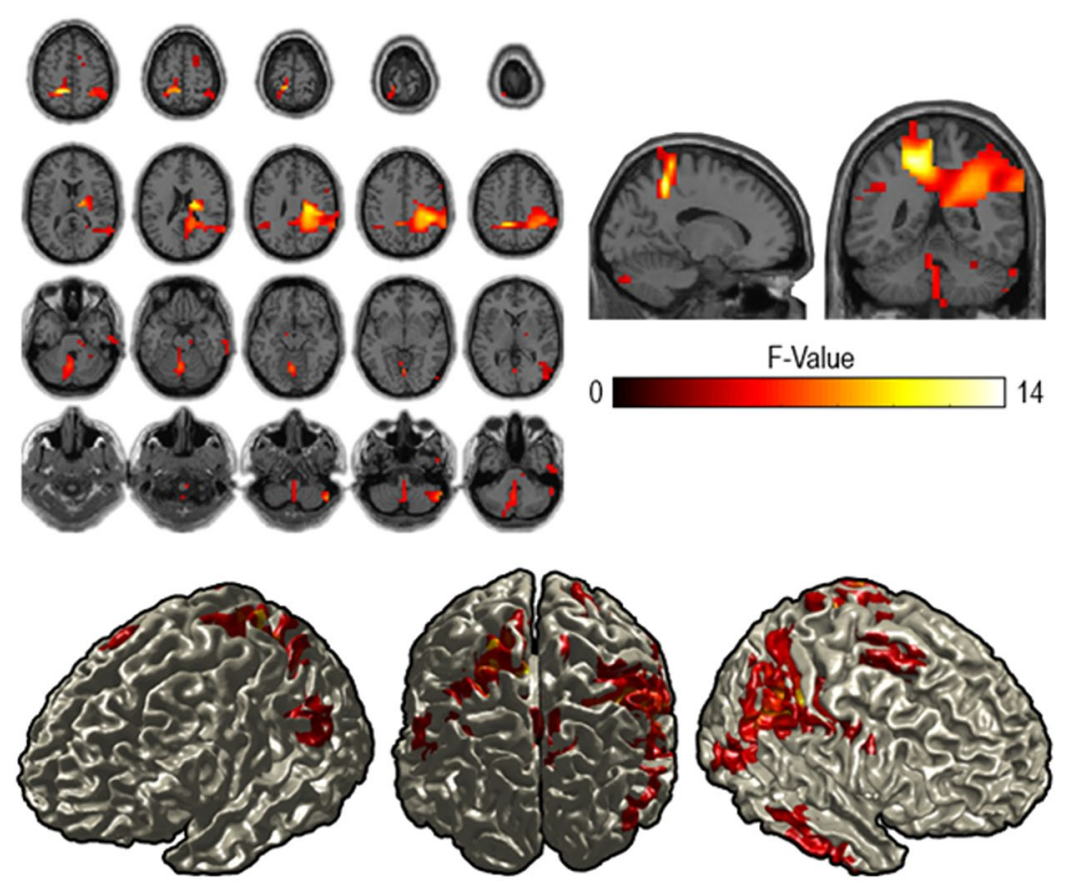

Figure 5. Reconstruction of the effect of context constraints in the alpha range $(8-12 \mathrm{~Hz})$. The source statistics reveal that the effect of the context constraint manipulation is most pronounced over left and right parietal regions. Upper figures show horizontal and sagittal as well as coronal slices for the alpha frequency band. Lower figures represent surface plots of the source statistics (F-values are thresholded at $p<0.05$ and controlled for multiple comparisons using cluster-based permutation tests).

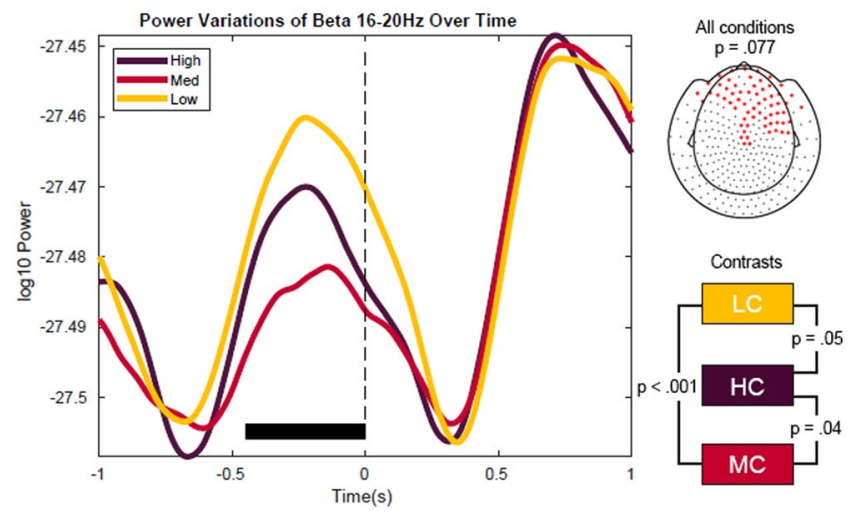

Figure 6. Beta power fluctuations as a function of context constraints as averaged across channel clusters. The striped line marks target word onset. The black horizontal bar indicates the time window of the cluster $[-500$ $\mathrm{ms}, 0 \mathrm{~ms}$ ]. The right topography shows the sensors of the cluster. The effect of context constrains (which does not reach significance threshold) is most pronounced over frontal sensors. Over these sensors, beta power showed the strongest decrease for the MC condition, followed by $\mathrm{HC}$ and LC.

predictability accounts ${ }^{11}$. In contrast, the pre-stimulus alpha/beta power fluctuations do not linearly relate to the degree of predictability of the target word given its sentential context. Our findings partially replicate previous results, showing that high context constraints induced a stronger alpha/beta power decrease than low constraint $^{21,23,25}$. Yet, the stronger alpha/beta power decrease for medium context constraints suggest that alpha and beta power does not reflect the degree of predictability of the target word given its context. Furthermore, while we initially defined the alpha frequency band between $8-12 \mathrm{~Hz}$ as in previous reports ${ }^{21,32,38,50}$, post-hoc analysis show that the strongest effects of context constraint were observed in the lower alpha range around $8 \mathrm{~Hz}$. This suggests that alpha effects previously linked to sentential constraint processing ${ }^{25}$ may be more prominently observed in the lower alpha range. The interpretation of the results could still be framed within the general mechanistic account of alpha being indicative of neuronal engagement ${ }^{38}$, considering that lower alpha band activity in the $7-9 \mathrm{~Hz}$ has been linked to information gating regarding visual processing ${ }^{51,52}$. In addition to the differences in amplitude fluctuations between pre-stimulus alpha and beta oscillations and post-stimulus M400, our effects exhibit distinct 

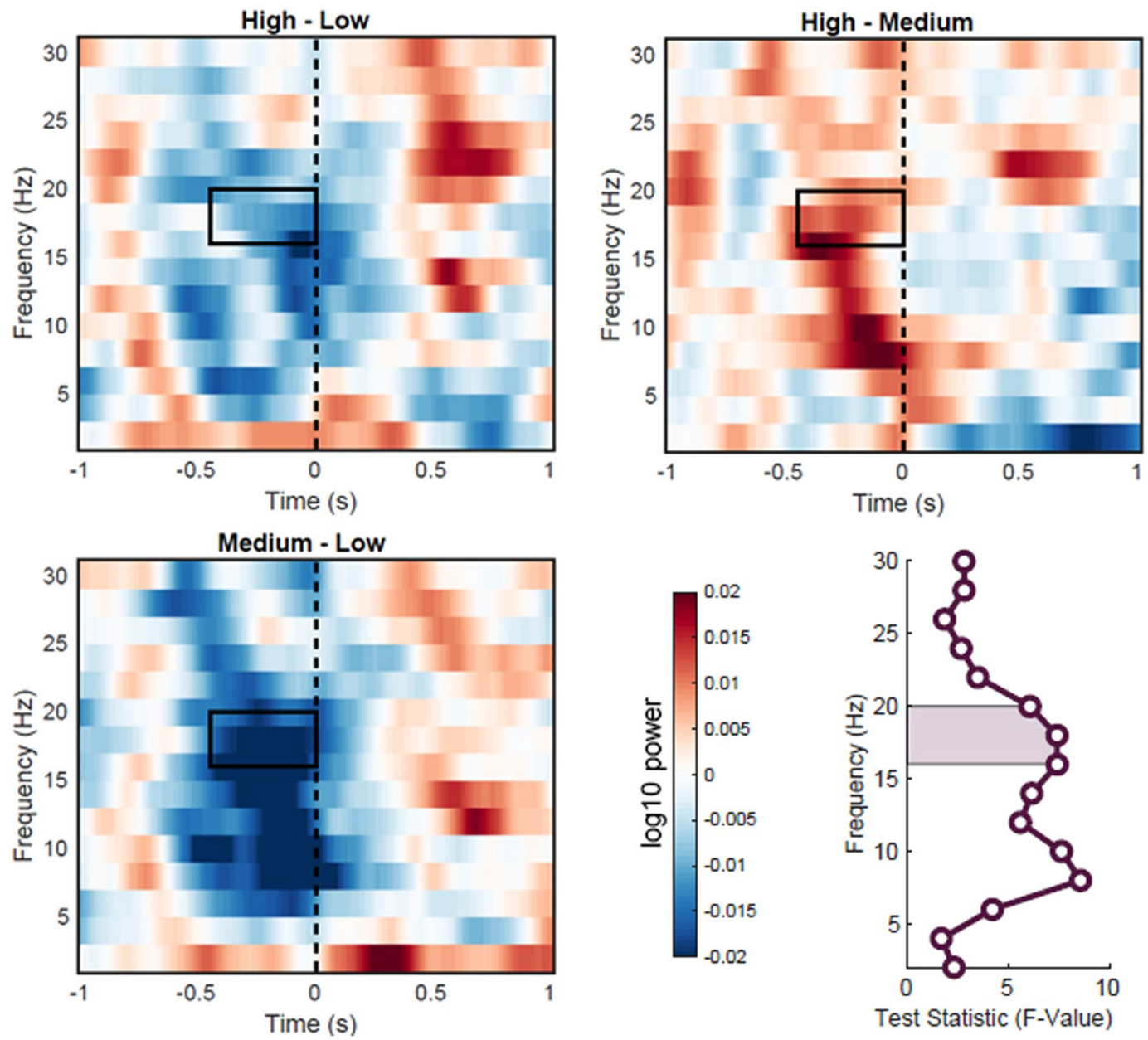

Figure 7. Time-frequency power contrasts between context constraint conditions within the beta band sensor cluster. Time-frequency plots are averaged across sensors that were identified by the cluster-based permutation statistics of the beta frequency band $(16-20 \mathrm{~Hz})$. The dashed line marks the onset of the target word. The rectangle indicates the time-window of the respective cluster, and the beta frequency band. Lower right figure shows the $\mathrm{F}$ statistics of the main effect of context constraints across frequencies, averaged within the time window [ $-500 \mathrm{~ms}, 0 \mathrm{~ms}$. The area under the curve shows the pre-defined beta frequency range used for clusterbased analysis $(16-20 \mathrm{~Hz}$ ). For this cluster (over frontal sensors) the effect of context constraint is strongest in the beta range $(16-18 \mathrm{~Hz})$ and in the lower alpha range $(8-10 \mathrm{~Hz})$.

topographical properties. The sources of the M400 have previously been localized to temporal as well as prefrontal areas, with a stronger prominence in the left hemisphere (see Lau and colleagues ${ }^{53}$ ). The topography of the M400 results in the present study is in line with these common findings. In contrast, the sources of the pre-stimulus alpha and beta power changes cover distinct areas. Alpha power modulations were most pronounced over parietal areas, and beta power effects were most pronounced over dorsolateral prefrontal areas. While alpha and beta power reacted similarly to the amount of context constrain within a sentence, the source reconstruction of these effects suggest that alpha power modulations and beta power effects do not reflect the same process. These results capture different alpha and beta dynamics that were reported in studies contrasting low and high sentence context constraints processing only ${ }^{25,39}$. These studies located the alpha and beta power differences predominantly over left inferior frontal areas and left middle and posterior temporal regions.

Pre-stimulus alpha and beta power modulations are not consistent with processes that relate to predictability. However, their dynamics and topographies could speculatively indicate that they relate to attention and/or working memory operations taking place during sentence processing ${ }^{39}$. Alpha oscillations have been previously related to attentional gating and the maintenance of task relevant items in working memory ${ }^{54-60}$. Parietal alpha power is shown to decrease with working memory load during encoding ${ }^{58,61,62}$, and may reflect the relative engagement of networks involved in the encoding of items to keep in memory, and the active inhibition of task irrelevant items ${ }^{56}$. The dorsolateral prefrontal cortex is a crucial region recruited for working memory operations ${ }^{63-65}$. The beta activity reported in our study could potentially reflect working memory load processing capacity ${ }^{66}$. This mechanism is crucial during sentence processing, where pre-stimulus alpha and beta oscillations could be then involved in the preselection and maintenance of lexical candidates ${ }^{39}$ (see also Piai and colleagues ${ }^{67}$ ). The set of target candidates that need to be retrieved and maintained should in particular be guided by semantic context ${ }^{68}$. 


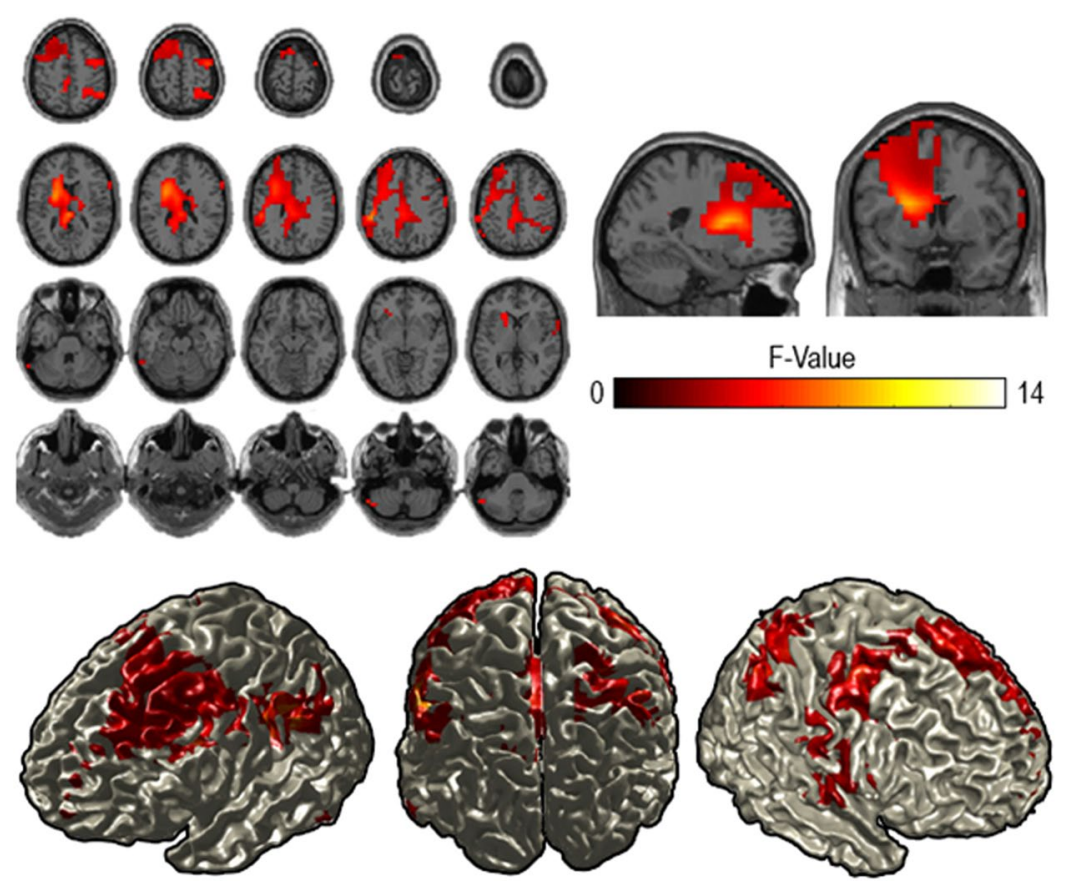

Figure 8. Reconstruction of the effect of context constraints in the beta range (16-20 Hz). The source statistics reveal that the effect of the context constraint manipulation is most pronounced over left and right dorsolateral prefrontal regions and parietal regions. Upper figures show horizontal and sagittal as well as coronal slices for the beta frequency band. Lower figures represent surface plots of the source statistics (F-values are thresholded at $\mathrm{p}<0.05$ and controlled for multiple comparisons using cluster-based permutation tests).

We speculate that the amount of possible lexical items to be encoded and maintained might differ depending on the degree of constraint, which would lead to distinct alpha and beta power modulations before target word onset. Compared to low or high context constraining sentences, intermediate context constraints may generate the highest competition between lexical candidates. In the high context constraining conditions, few items are competing, which would require low working memory demands. In low context constraining conditions, the sentential context is broad enough that the number of alternative candidates for sentence ending would be much higher than working memory capacities, resulting in working memory processes being disengaged in this setting. Eventually, in medium context constraining settings, more distractors are to be maintained in working memory than in other context constrains conditions, which in turn is reflected by the stronger alpha power decrease.

As an alternative explanation, the changes in alpha and beta activity could also reflect an overall change of alertness or attentional lapses as a function of the predictability of the stimulus ${ }^{61,69}$. In these studies, participants who had higher alpha power at the presentation of a relevant sentential context were more likely to discard this sentential information for incoming linguistic processing. A decrease in alpha power prior to target word onset could thus potentially reflect general higher levels of alertness that would affect the processing of relevant contextual words. However, we do not think that changes in alertness can explain our results as we controlled our design to result in similar levels of alertness across conditions by randomizing the presentation of the sentences and matching linguistic features like word frequency and length.

In sum, using sentences with different context constraints that are matched on other linguistic variables like lexical frequency and word length, this MEG study shows that pre-stimulus alpha/beta power in the course of the sentence is modulated by context constraints. However, the alpha/beta power decrease is strongest for medium constraining sentences, which defies previous interpretations of this marker in light of a prediction mechanism. The non-monotonic sensitivity of the alpha/beta power fluctuations to these different levels of constraints highlight the importance of including intermediate conditions in language research. Therefore, our results do not support the hypothesis that alpha and beta oscillatory markers reflect linguistic predictability, and a mechanistic account relating alpha/beta oscillations and the degree of sentence context constraint remains to be elaborated.

\section{Data availability}

The datasets generated during and/or analyzed during the current study are available in the Donders repository, https://data.donders.ru.nl/collections/di/dccn/DSC_3011097.01_968?4.

Received: 15 July 2019; Accepted: 4 December 2019;

Published online: 27 December 2019 


\section{References}

1. Cairns, H. S., Cowart, W. \& Jablon, A. D. Effects of prior context upon the integration of lexical information during sentence processing. J. Verbal Learn. Verbal Behav. 20, 445-453 (1981).

2. Glucksberg, S., Kreuz, R. J. \& Rho, S. H. Context can constrain lexical access: Implications for models of language comprehension. J. Exp. Psychol. Learn. Mem. Cogn. 12, 323 (1986).

3. Hagoort, P. Chapter 28 - MUC (Memory, Unification, Control): A Model on the Neurobiology of Language Beyond Single Word Processing. In Neurobiology of Language (eds. Hickok, G. \& Small, S. L.) 339-347 (Academic Press). https://doi.org/10.1016/B9780-12-407794-2.00028-6.(2016)

4. Morris, R. K. Lexical and message-level sentence context effects on fixation times in reading. J. Exp. Psychol. Learn. Mem. Cogn. 20, 92-103 (1994).

5. Moss, H. E. \& Marslen-Wilson, W. D. Access to word meanings during spoken language comprehension: Effects of sentential semantic context. J. Exp. Psychol. Learn. Mem. Cogn. 19, 1254-1276 (1993).

6. Rommers, J., Dijkstra, T. \& Bastiaansen, M. Context-dependent Semantic Processing in the Human. Brain: Evidence from Idiom Comprehension. J. Cogn. Neurosci. 25, 762-776 (2012).

7. Federmeier, K. D. Thinking ahead: The role and roots of prediction in language comprehension. Psychophysiology 44, 491-505 (2007).

8. Frank, S. L. \& Willems, R. M. Word predictability and semantic similarity show distinct patterns of brain activity during language comprehension. Lang. Cogn. Neurosci. 32, 1192-1203 (2017).

9. Xu, J., Kemeny, S., Park, G., Frattali, C. \& Braun, A. Language in context: emergent features of word, sentence, and narrative comprehension. NeuroImage 25, 1002-1015 (2005).

10. Janssen, E. M., van der Ven, S. H. G., van Hoogmoed, A. H. \& Leseman, P. P. M. The effect of anticipated achievement feedback on students' semantic processing as indicated by the N400 cloze effect. Learn. Instr. 47, 80-90 (2017).

11. Kutas, M. \& Federmeier, K. D. Thirty years and counting: Finding meaning in the N400 component of the event related brain potential (ERP). Annu. Rev. Psychol. 62, 621-647 (2011).

12. Tromp, J., Peeters, D., Meyer, A. S. \& Hagoort, P. The combined use of virtual reality and EEG to study language processing in naturalistic environments. Behav. Res. Methods 50, 862-869 (2018).

13. Frank, S. L., Otten, L. J., Galli, G. \& Vigliocco, G. The ERP response to the amount of information conveyed by words in sentences. Brain Lang. 140, 1-11 (2015).

14. DeLong, K. A., Urbach, T. P. \& Kutas, M. Probabilistic word pre-activation during language comprehension inferred from electrical brain activity. Nat. Neurosci. 8, 1117 (2005).

15. Diaz, M. T. \& Swaab, T. Y. Electrophysiological differentiation of phonological and semantic integration in word and sentence contexts. Brain Res. 1146, 85-100 (2007).

16. Freunberger, D. \& Roehm, D. The costs of being certain: Brain potential evidence for linguistic preactivation in sentence processing. Psychophysiology 54, 824-832 (2017).

17. Ito, A., Corley, M., Pickering, M. J., Martin, A. E. \& Nieuwland, M. S. Predicting form and meaning: Evidence from brain potentials. J. Mem. Lang. 86, 157-171 (2016).

18. Van Petten, C. \& Luka, B. J. Prediction during language comprehension: Benefits, costs, and ERP components. Int. J. Psychophysiol. 83, 176-190 (2012).

19. Kuperberg, G. R. \& Jaeger, T. F. What do we mean by prediction in language comprehension? Lang. Cogn. Neurosci. 31, 32-59 (2016).

20. Molinaro, N., Monsalve, I. F. \& Lizarazu, M. Is there a common oscillatory brain mechanism for producing and predicting language? Lang. Cogn. Neurosci. 31, 145-158 (2016).

21. Rommers, J., Dickson, D. S., Norton, J. J. S., Wlotko, E. W. \& Federmeier, K. D. Alpha and theta band dynamics related to sentential constraint and word expectancy. Lang. Cogn. Neurosci. 32, 576-589 (2017).

22. Molinaro, N., Barraza, P. \& Carreiras, M. Long-range neural synchronization supports fast and efficient reading: EEG correlates of processing expected words in sentences. NeuroImage 72, (2013).

23. Lewis, A. G., Wang, L. \& Bastiaansen, M. Fast oscillatory dynamics during language comprehension: Unification versus maintenance and prediction? Brain Lang. 148, 51-63 (2015).

24. Wang, L., Zhu, Z. \& Bastiaansen, M. Integration or Predictability? A Further Specification of the Functional Role of Gamma Oscillations in Language Comprehension. Front. Psychol. 3, (2012).

25. Wang, L., Hagoort, P. \& Jensen, O. Language Prediction Is Reflected by Coupling between Frontal Gamma and Posterior Alpha Oscillations. J. Cogn. Neurosci. 30, 432-447 (2017).

26. Mamashli, F., Khan, S., Obleser, J., Friederici, A. D. \& Maess, B. Oscillatory dynamics of cortical functional connections in semantic prediction. Hum. Brain Mapp. 40, 1856-1866 (2019).

27. Arnal, L. H. \& Giraud, A.-L. Cortical oscillations and sensory predictions. Trends Cogn. Sci. 16, 390-398 (2012).

28. Lewis, A. G., Schoffelen, J.-M., Schriefers, H. \& Bastiaansen, M. A Predictive Coding Perspective on Beta Oscillations during Sentence-Level Language Comprehension. Front. Hum. Neurosci. 10 (2016).

29. Bastos, A. M. et al. Visual Areas Exert Feedforward and Feedback Influences through Distinct Frequency Channels. Neuron 85, 390-401 (2015)

30. Bonnefond, M., Kastner, S. \& Jensen, O. Communication between Brain Areas Based on Nested Oscillations. eNeuro 4 (2017).

31. Fries, P. Rhythms For Cognition: Communication Through Coherence. Neuron 88, 220-235 (2015).

32. Lam, N. H. L., Schoffelen, J.-M., Uddén, J., Hultén, A. \& Hagoort, P. Neural activity during sentence processing as reflected in theta, alpha, beta, and gamma oscillations. NeuroImage 142, 43-54 (2016).

33. Piai, V., Rommers, J. \& Knight, R. T. Evidence for a causal link between left posterior alpha-beta power decreases and context-driven word production. bioRxiv 150748 https://doi.org/10.1101/150748 (2017).

34. Bastiaansen, M. \& Hagoort, P. Frequency-based Segregation of Syntactic and Semantic Unification during Online Sentence Level Language Comprehension. J. Cogn. Neurosci. 27, 2095-2107 (2015).

35. Wang, L. et al. Beta oscillations relate to the N400m during language comprehension. Hum. Brain Mapp. 33, 2898-2912 (2012).

36. Weiss, S. \& Mueller, H. M. “Too Many betas do not Spoil the Broth”: The Role of Beta Brain Oscillations in Language Processing. Front. Psychol. 3 (2012).

37. Willems, R. M., Oostenveld, R. \& Hagoort, P. Early decreases in alpha and gamma band power distinguish linguistic from visual information during spoken sentence comprehension. Brain Res. 1219, 78-90 (2008).

38. Jensen, O. \& Mazaheri, A. Shaping Functional Architecture by Oscillatory Alpha Activity: Gating by Inhibition. Front. Hum. Neurosci. 4, (2010).

39. Piai, V., Roelofs, A., Rommers, J. \& Maris, E. Beta oscillations reflect memory and motor aspects of spoken word production. Hum. Brain Mapp. 36, 2767-2780 (2015).

40. Keuleers, E., Brysbaert, M. \& New, B. SUBTLEX-NL: A new measure for Dutch word frequency based on film subtitles. Behav. Res. Methods 42, 643-650 (2010).

41. Stolk, A., Todorovic, A., Schoffelen, J.-M. \& Oostenveld, R. Online and offline tools for head movement compensation in MEG. NeuroImage 68, 39-48 (2013). 
42. Oostenveld, R., Fries, P., Maris, E. \& Schoffelen, J.-M. FieldTrip: Open Source Software for Advanced Analysis of MEG, EEG, and Invasive Electrophysiological Data. Comput. Intell. Neurosci. 2011 (2011).

43. Hyvärinen, A. \& Oja, E. Independent component analysis: algorithms and applications. Neural Netw. 13, 411-430 (2000).

44. Halgren, E. et al. N400-like Magnetoencephalography Responses Modulated by Semantic Context, Word Frequency, and Lexical Class in Sentences. NeuroImage 17, 1101-1116 (2002).

45. Lau, E., Almeida, D., Hines, P. C. \& Poeppel, D. A lexical basis for N400 context effects: Evidence from MEG. Brain Lang. 111, 161-172 (2009)

46. Bastiaansen, M. C. M. \& Knösche, T. R. Tangential derivative mapping of axial MEG applied to event-related desynchronization research. Clin. Neurophysiol. 111, 1300-1305 (2000).

47. Gross, J. et al. Dynamic imaging of coherent sources: Studying neural interactions in the human brain. Proc. Natl. Acad. Sci. 98, 694-699 (2001)

48. Nolte, G. The magnetic lead field theorem in the quasi-static approximation and its use for magnetoencephalography forward calculation in realistic volume conductors. Phys. Med. Biol. 48, 3637-3652 (2003).

49. Maris, E. \& Oostenveld, R. Nonparametric statistical testing of EEG- and MEG-data. J. Neurosci. Methods 164, 177-190 (2007).

50. Mathewson, K. E., Gratton, G., Fabiani, M., Beck, D. M. \& Ro, T. To See or Not to See: Prestimulus $\alpha$ Phase Predicts Visual Awareness. J. Neurosci. 29, 2725-2732 (2009).

51. Busch, N. A., VanRullen, R. \& Spontaneous, E. E. G. oscillations reveal periodic sampling of visual attention. Proc. Natl. Acad. Sci. 107, 16048-16053 (2010).

52. Hanslmayr, S., Volberg, G., Wimber, M., Dalal, S. S. \& Greenlee, M. W. Prestimulus Oscillatory Phase at 7 Hz Gates Cortical Information Flow and Visual Perception. Curr. Biol. 23, 2273-2278 (2013).

53. Lau, E. F., Phillips, C. \& Poeppel, D. A cortical network for semantics: (de)constructing the N400. Nat. Rev. Neurosci. 9, 920-933 (2008).

54. Hanslmayr, S., Staudigl, T. \& Fellner, M.-C. Oscillatory power decreases and long-term memory: the information via desynchronization hypothesis. Front. Hum. Neurosci. 6 (2012).

55. Röhm, D., Klimesch, W., Haider, H. \& Doppelmayr, M. The role of theta and alpha oscillations for language comprehension in the human electroencephalogram. Neurosci. Lett. 310, 137-140 (2001).

56. Roux, F. \& Uhlhaas, P. J. Working memory and neural oscillations: alpha-gamma versus theta-gamma codes for distinct WM information? Trends Cogn. Sci. 18, 16-25 (2014).

57. Bastiaansen, M. C. M., Posthuma, D., Groot, P. F. C. \& de Geus, E. J. C. Event-related alpha and theta responses in a visuo-spatial working memory task. Clin. Neurophysiol. 113, 1882-1893 (2002).

58. Jensen, O. \& Tesche, C. D. Frontal theta activity in humans increases with memory load in a working memory task. Eur. J. Neurosci. 15, 1395-1399 (2002).

59. Piai, V. et al. Direct brain recordings reveal hippocampal rhythm underpinnings of language processing. Proc. Natl. Acad. Sci. 113, 11366-11371 (2016)

60. Bastiaansen, M. \& Hagoort, P. Oscillatory neuronal dynamics during language comprehension. In Progress in Brain Research (eds. Neuper, C. \& Klimesch, W.) vol. 159 179-196 (Elsevier, 2006).

61. Boudewyn, M. A. et al. Sensitivity to Referential Ambiguity in Discourse: The Role of Attention, Working Memory, and Verbal Ability. J. Cogn. Neurosci. 27, 2309-2323 (2015).

62. Sauseng, P., Klimesch, W., Schabus, M. \& Doppelmayr, M. Fronto-parietal EEG coherence in theta and upper alpha reflect central executive functions of working memory. Int. J. Psychophysiol. 57, 97-103 (2005).

63. Barbey, A. K., Koenigs, M. \& Grafman, J. Dorsolateral Prefrontal Contributions to Human Working Memory. Cortex J. Devoted Study Nerv. Syst. Behav. 49, 1195-1205 (2013).

64. Duncan, J. \& Owen, A. M. Common regions of the human frontal lobe recruited by diverse cognitive demands. Trends Neurosci. 23, 475-483 (2000).

65. Duncan, J. The multiple-demand (MD) system of the primate brain: mental programs for intelligent behaviour. Trends Cogn. Sci. 14, $172-179$ (2010).

66. Yoon, J. H., Grandelis, A. \& Maddock, R. J. Dorsolateral Prefrontal Cortex GABA Concentration in Humans Predicts Working Memory Load Processing Capacity. J. Neurosci. 36, 11788-11794 (2016).

67. Piai, V., Roelofs, A. \& Maris, E. Oscillatory brain responses in spoken word production reflect lexical frequency and sentential constraint. Neuropsychologia 53, 146-156 (2014).

68. Levelt, W. J. M. Speaking: From Intention to Articulation. (MIT Press, 1993).

69. Boudewyn, M. A. \& Carter, C. S. I must have missed that: Alpha-band oscillations track attention to spoken language. Neuropsychologia 117, 148-155 (2018)

\section{Author contributions}

All authors contributed to study concept and design. Data collection, analysis and interpretation were performed by René Terporten, data analysis was supported by Bohan Dai. Project execution, development and data interpretation were supervised by Anne Kösem and Peter Hagoort. Data analysis was supervised by Anne Kösem and Jan-Mathijs Schoffelen. René Terporten wrote the manuscript and Anne Kösem, Jan-Mathijs Schoffelen and Peter Hagoort provided critical revision. All authors approved the final version of the manuscript for submission.

\section{Competing interests}

The authors declare no competing interests.

\section{Additional information}

Correspondence and requests for materials should be addressed to R.T.

Reprints and permissions information is available at www.nature.com/reprints.

Publisher's note Springer Nature remains neutral with regard to jurisdictional claims in published maps and institutional affiliations. 
(c) (i) Open Access This article is licensed under a Creative Commons Attribution 4.0 International License, which permits use, sharing, adaptation, distribution and reproduction in any medium or format, as long as you give appropriate credit to the original author(s) and the source, provide a link to the Creative Commons license, and indicate if changes were made. The images or other third party material in this article are included in the article's Creative Commons license, unless indicated otherwise in a credit line to the material. If material is not included in the article's Creative Commons license and your intended use is not permitted by statutory regulation or exceeds the permitted use, you will need to obtain permission directly from the copyright holder. To view a copy of this license, visit http://creativecommons.org/licenses/by/4.0/.

(C) The Author(s) 2019 